\title{
GPR Investigations of the Roman Baths at Carsulae, Italy
}

\section{Donald M. Thieme (VSU) and Bruce Brown (Australian National University) VALDOSTA STATE UNIVERSITY}

\section{Carsulae}

- In Italy north of Rome (Umbria)

- Founded ca 300 BCE

- Gained importance with building of Via Flaminia, 220-219 BCE

- Many works constructed under Caesar Augustus, 27 BCE - AD 14

- Arco di Troiana

- Forum

- Ampitheater/Theater

- Baths 

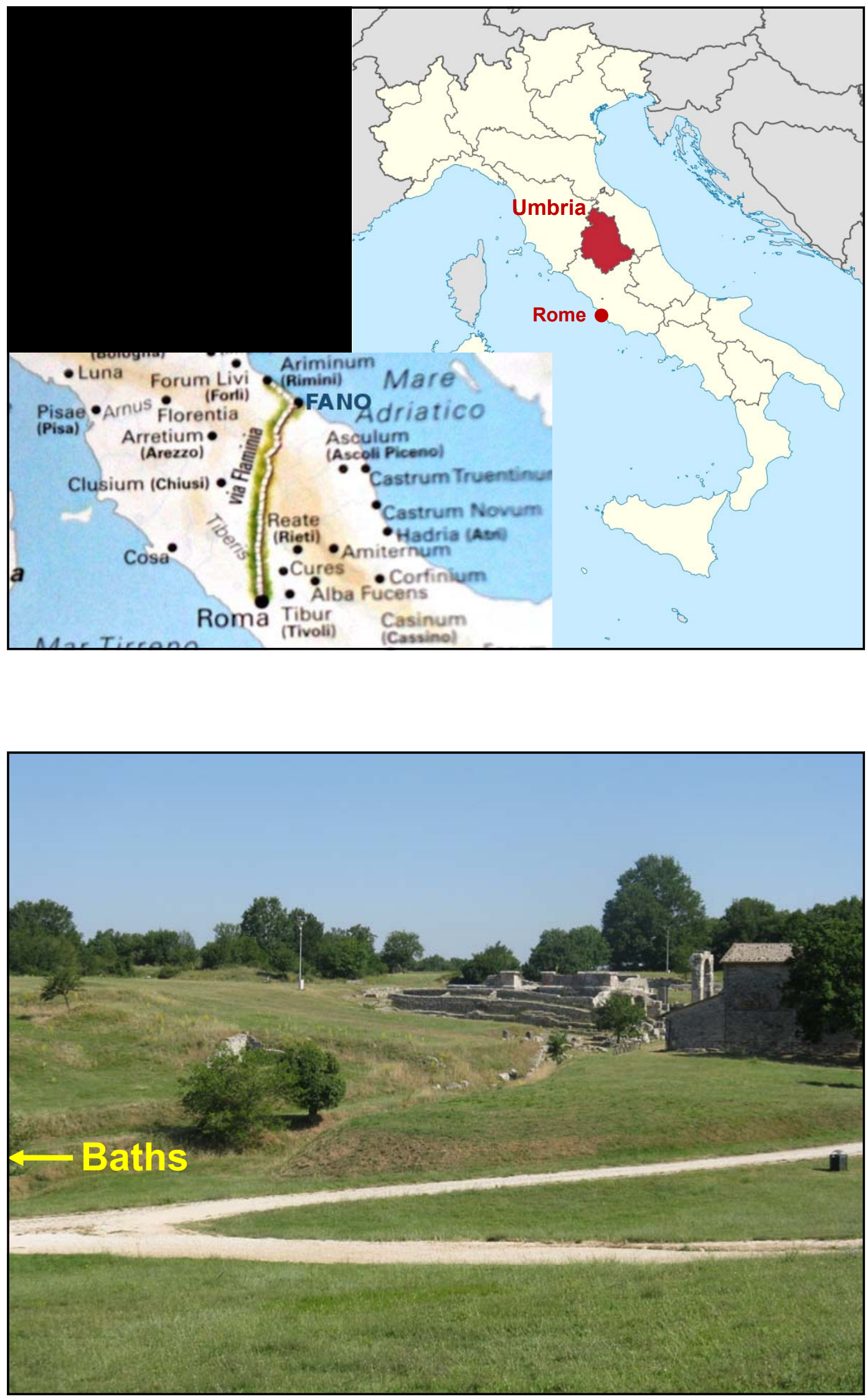

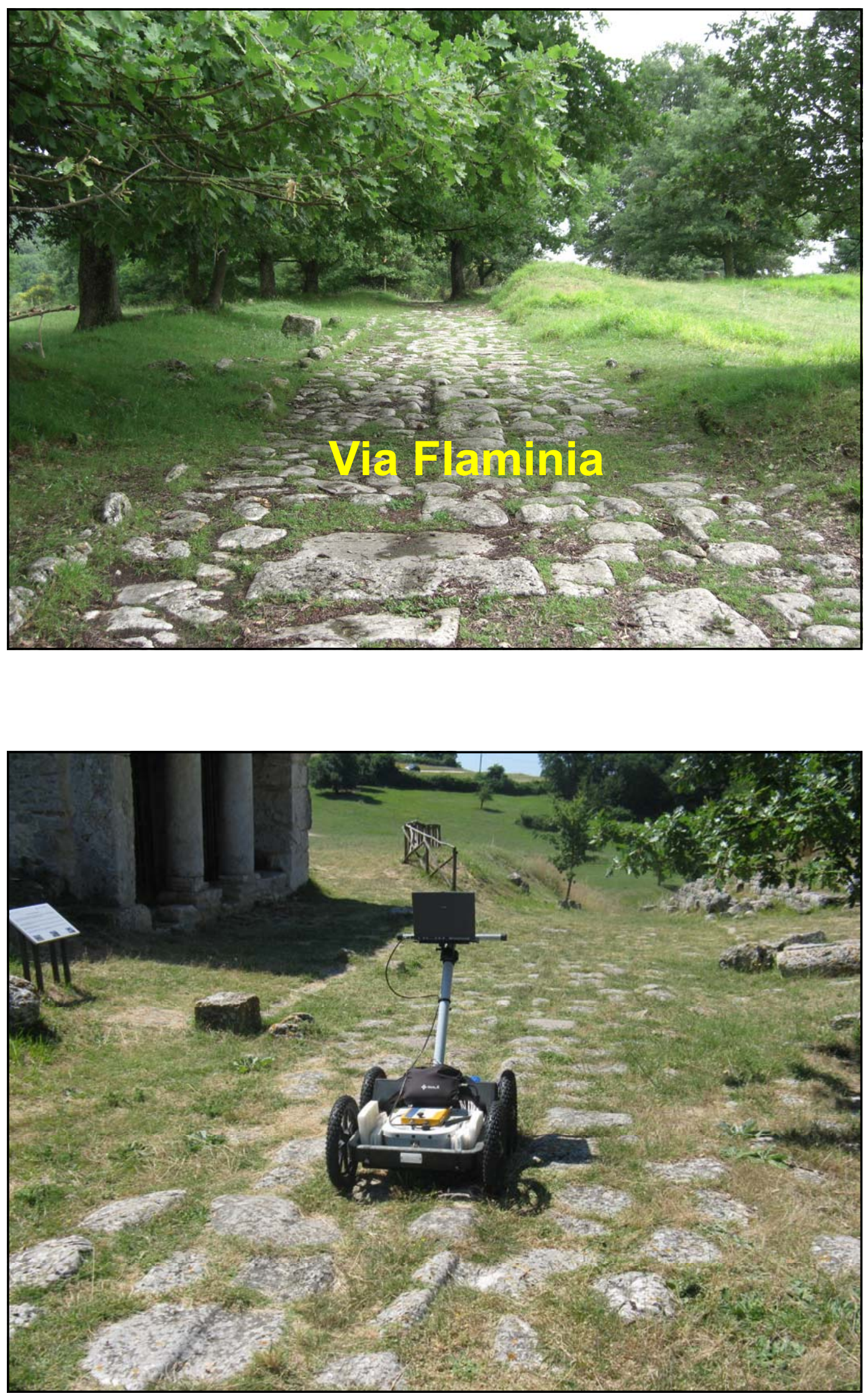


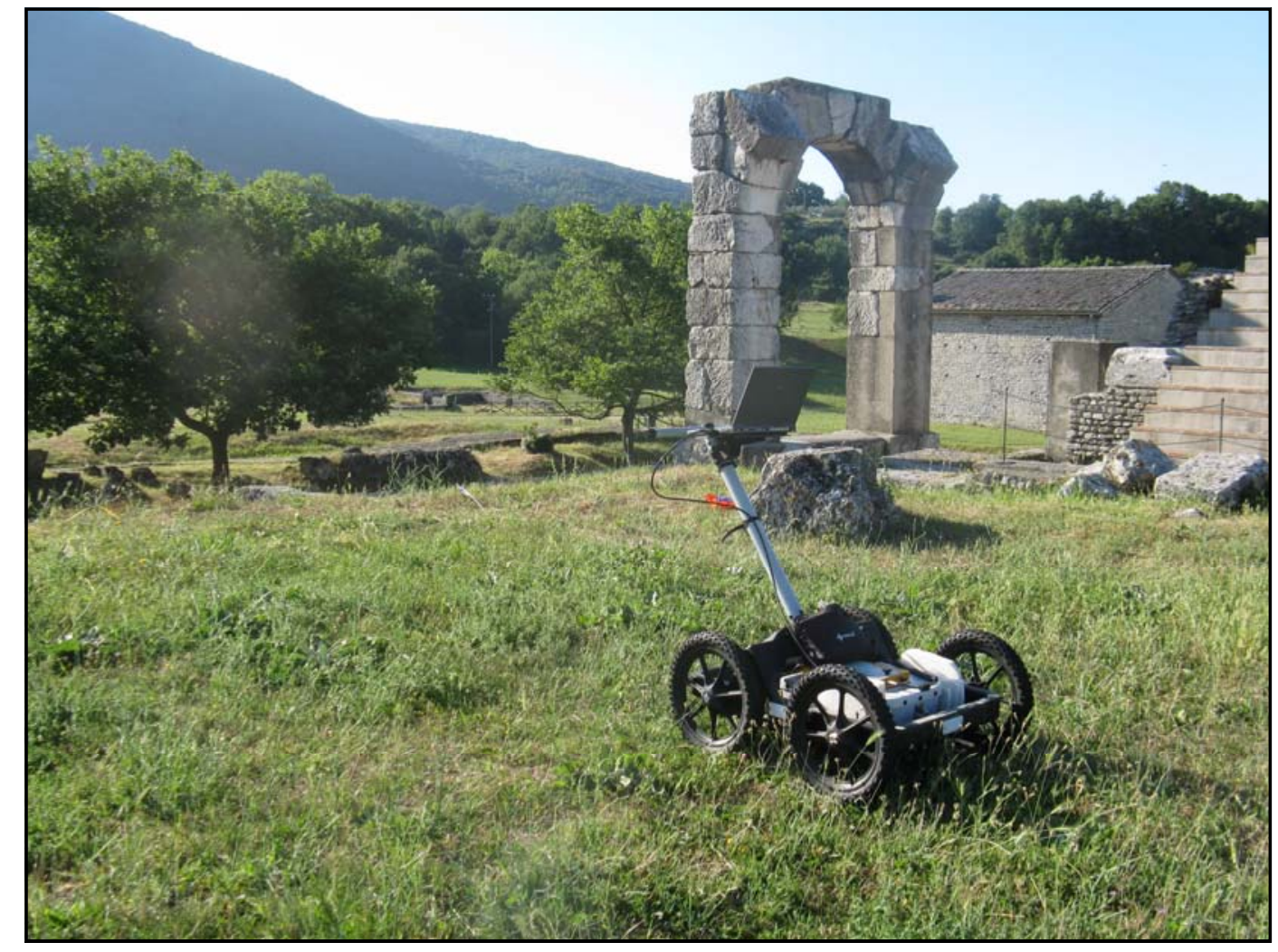

\section{Carsulae Roman Baths}

- Mapped and partially excavated along wih other Carsulae ruins by Umberto Ciotti, soprintendente for Umbria from 1950s to 1970s

- Excavations by Dr. Jane Whitehead (VSU) began in 2004

- Field Season with GPR in 2011, prior to

- Roof constructed over Baths (2012)

- Reconstruction of Bath architectural details begun in 2014. 

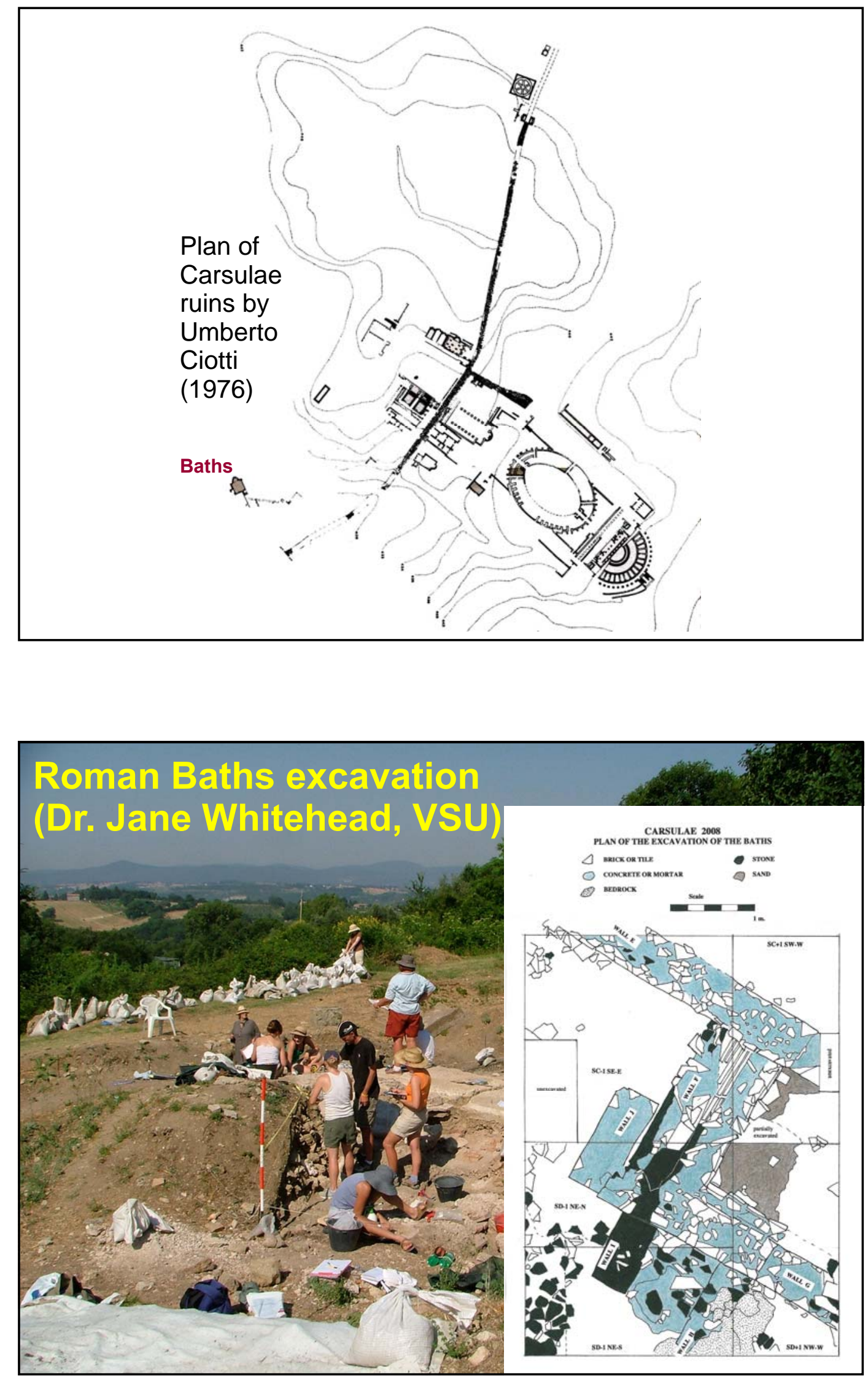


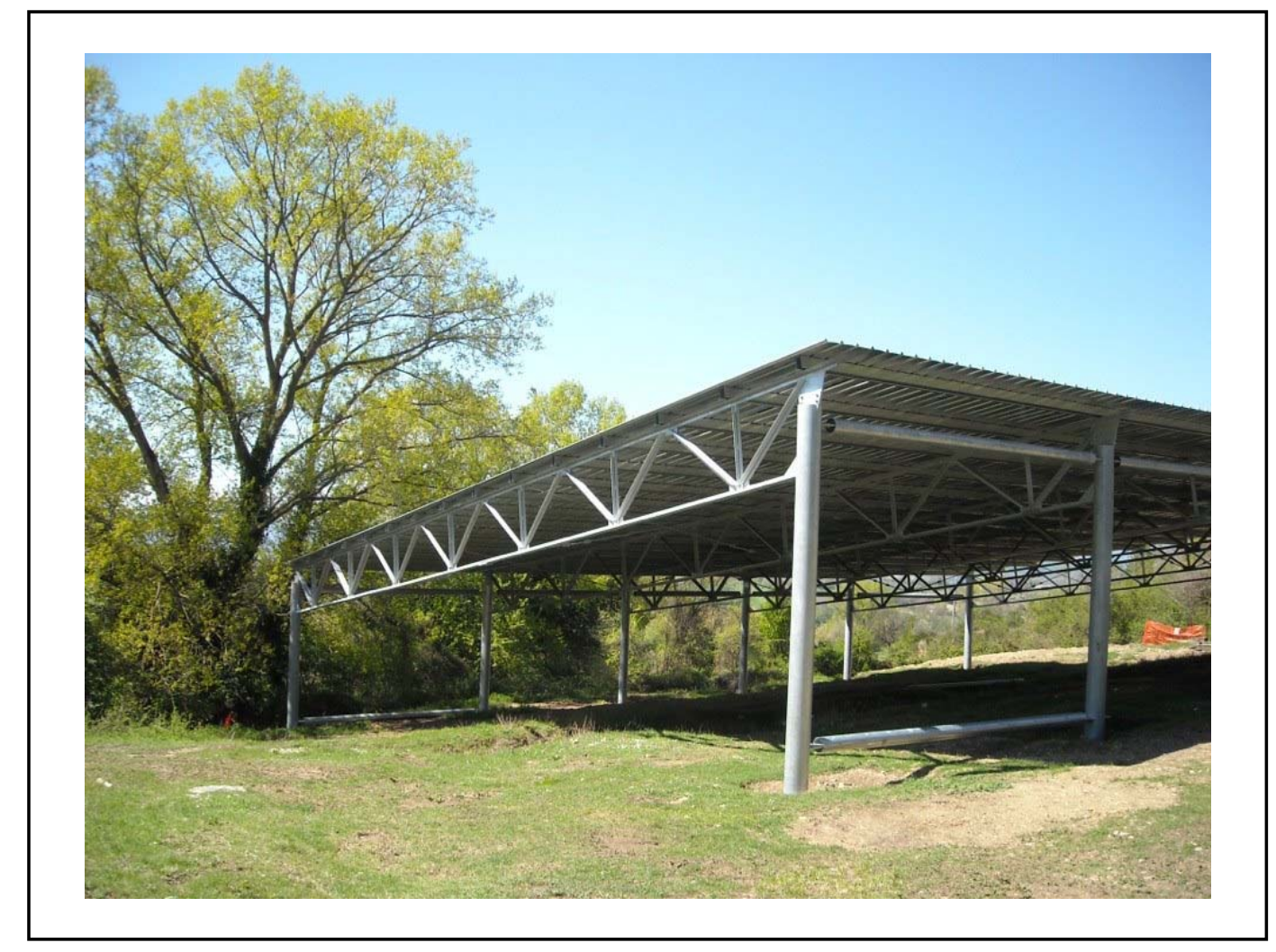

\section{GPR of Carsulae Baths}

- Thieme supervised fieldwork by project project participants

- June 13 - July 1, 2011

- Malå 500 MHz GPR system

- mounted on rough terrain cart

- GroundVision 2 software on an old HP Omnibook

- 16 grids of GPR data, 13 from Baths 


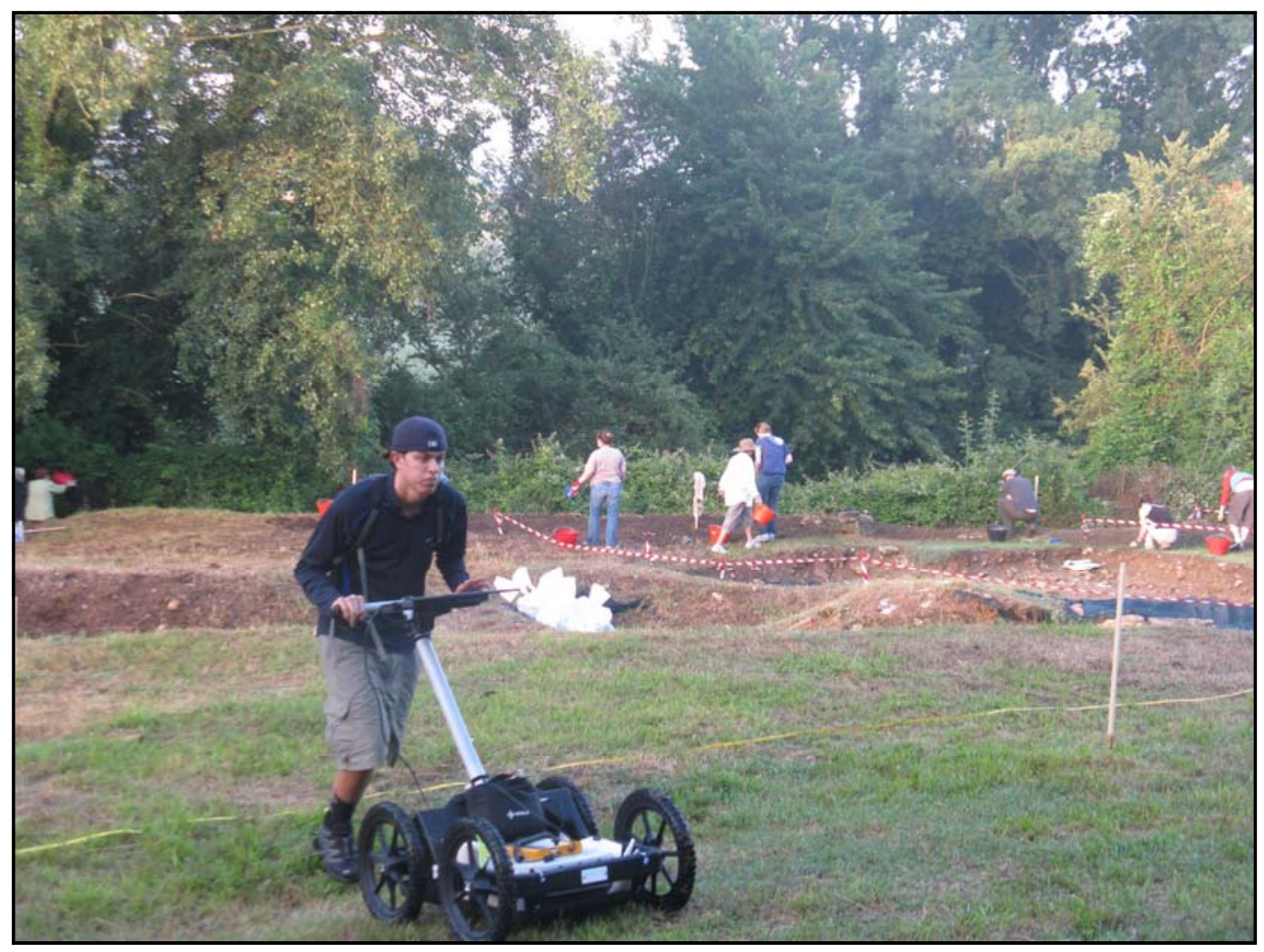

GRID

CarBath1

CarBath2

CarBath3

CarBath4

CarBath5

CarBath6

CarBath7

CarBath8

CarBath9

CarBath11

CarDol

CarForum1

CarForum2

CarForum3

CarShed1

CarShed2

CarShed5

CarBathNS

CarVia1

CarVia2

CarVia3

\begin{tabular}{c} 
Profiles \\
\hline 6 \\
19 \\
30 \\
21 \\
38 \\
12 \\
31 \\
25 \\
12 \\
21 \\
2 \\
3 \\
11 \\
12 \\
1 \\
1 \\
18 \\
1 \\
2 \\
15 \\
28
\end{tabular}

$$
\begin{aligned}
& \frac{\text { Direction }}{W \rightarrow E} \\
& E \rightarrow W \\
& S \rightarrow N \\
& E \rightarrow W \\
& S \rightarrow N \\
& S \rightarrow N \\
& E \rightarrow W \\
& S \rightarrow N \\
& S \rightarrow N \\
& W \rightarrow E \\
& N A \\
& E \rightarrow W \\
& E \rightarrow W \\
& N \rightarrow S \\
& E \rightarrow W \\
& E \rightarrow W \\
& N \rightarrow S \\
& S \rightarrow N \\
& W \rightarrow E \\
& N \rightarrow S \\
& S \rightarrow N
\end{aligned}
$$

\section{Began at}

$$
\text { NA }
$$

Lower Right

Lower Left

Lower Right

Lower Left

Lower Left

Lower Right

Lower Left

Lower Left

Lower Left

NA

Lower Left

Lower Left

Lower Left

NA

NA

Lower Left

NA

NA
Lower Left

Lower Left
Samp Freq

5118.8

5118.8

4993.9

5118.8

5118.8

5118.8

5118.8

5118.8

5118.8

5118.8

3011.0

5118.8

5118.8

5118.8

4993.9

5118.8

5118.8

5118.8

5118.8
\# Samp

452
130
222

222
130

128

202

202

202

202

208

300

202

202

202

456

530

80

210

350
TimWind (ns) Tracelnt (m)

$\begin{array}{ll}88.3 & 0.303 \\ 25.4 & 0.100\end{array}$

$\begin{array}{ll}44.5 & 0.017\end{array}$

$25.4 \quad 0.098$

$25.0 \quad 0.098$

$\begin{array}{ll}39.5 & 0.017\end{array}$

$39.5 \quad 0.017$

$39.5 \quad 0.017$

$\begin{array}{ll}39.5 & 0.017\end{array}$

$40.6 \quad 0.009$

$\begin{array}{ll}99.6 & 0.049\end{array}$

$39.5 \quad 0.009$

$39.5 \quad 0.009$

$\begin{array}{ll}39.5 & 0.009\end{array}$

$91.3 \quad 0.009$

$103.5 \quad 0.049$

15.6

0.017

$41.0 \quad 0.009$

$\begin{array}{ll}68.4 & 0.017\end{array}$ 


\section{GPR Data Analysis}

- Processing in San Gemini, 2011

- Conversion to *.dzt

- RadExplorer filtering for DC removal, Time Zero adjustment, Amplitude Correction

- Inconsistent Use of Bandpass Filters and Deconvolution

- GPR Process used to "time slice" CarBath2-9, CarBath11, CarForum2-3, CarVia2

\section{GPR Data Analysis}

- Processing by Bruce Brown, 2016-2017

- Thieme posted files for CarBath1-6 to

- "http://ww2.valdosta.edu/ dmthieme/Carsulae/ CarsulaeGPR.htm"

- GPR Process used to time slice these 6 grids

- Synthesis on common level of $\sim 10 \mathrm{~ns}$ (80 cm bs @ $8 \mathrm{~cm} / \mathrm{ns}$ )

- TIFF images floated over CAD output of excavation site plan 


\begin{tabular}{|ccc|}
\hline Material & RDP & Velocity $(\mathbf{c m} / \mathbf{n s})$ \\
\hline Air & 1 & 30 \\
\hline Ice & $3-4$ & 16 \\
\hline Fresh Water & 80 & 3 \\
\hline Salt Water & 80 & 1 \\
\hline Dry Sand & $3-5$ & 15 \\
\hline Wet Sand & $20-30$ & 6 \\
\hline Shale and Clay & $5-20$ & 8 \\
\hline Silt & $5-30$ & 7 \\
\hline Limestone & $4-8$ & 12 \\
\hline Granite & $4-6$ & 13 \\
\hline (Dry) Salt & $5-6$ & \\
We used a velocity of 8 cm/ns to estimate the depth \\
in the time slice maps for Carsulae. The limestone \\
rubble is conducting electricity a lot more rapidly than \\
is the overlying or underlying loose sediment.
\end{tabular}

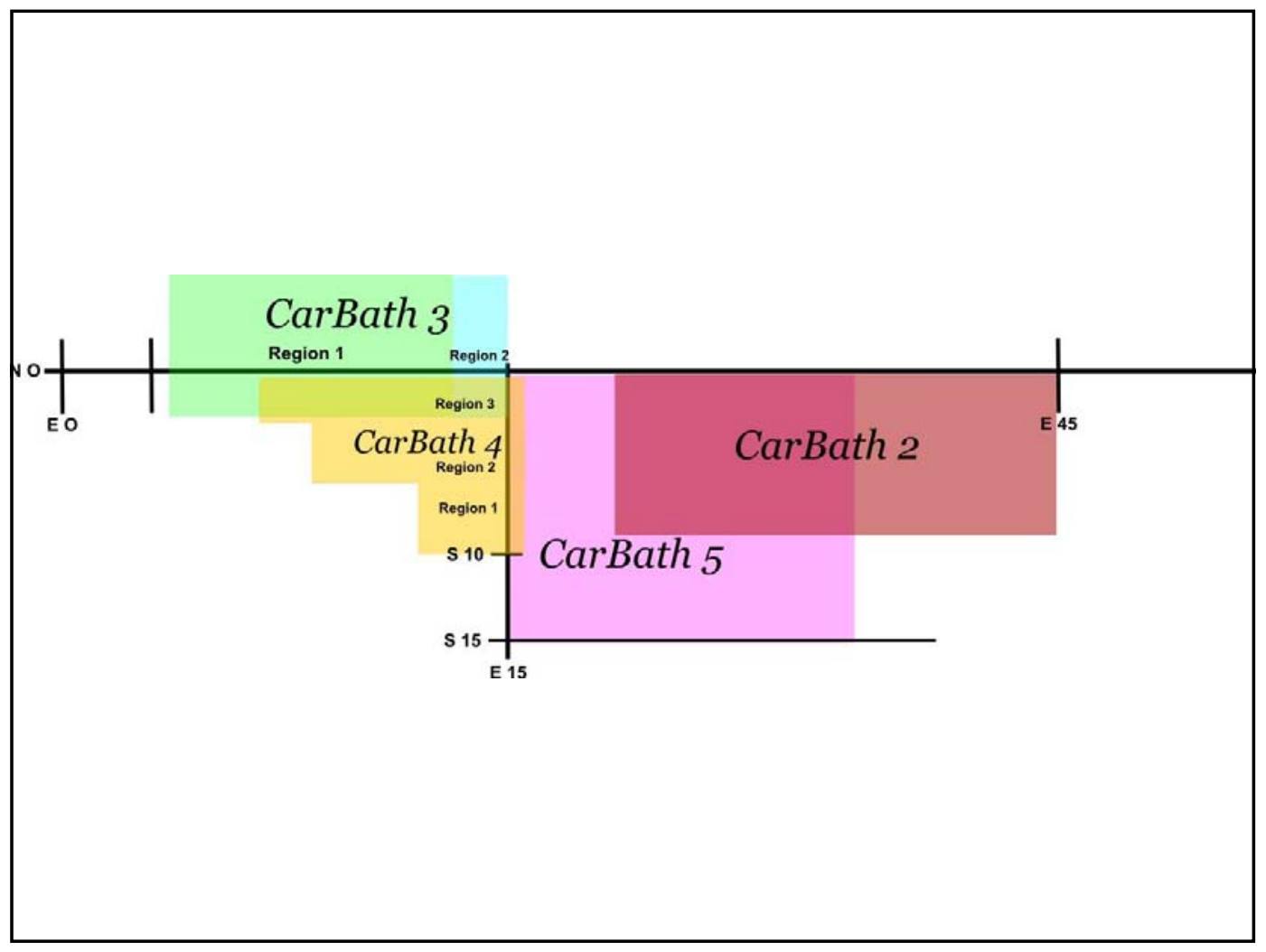



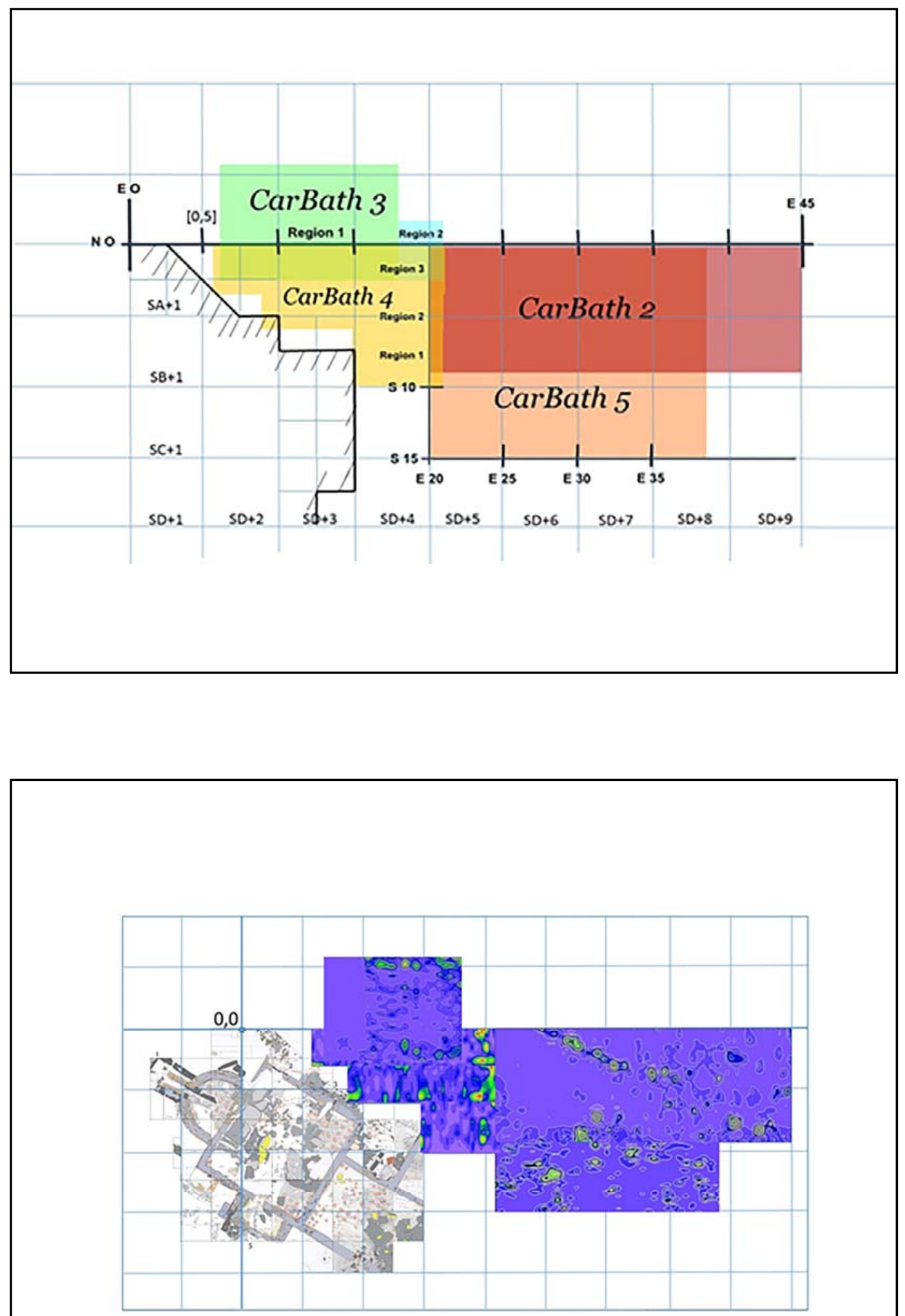


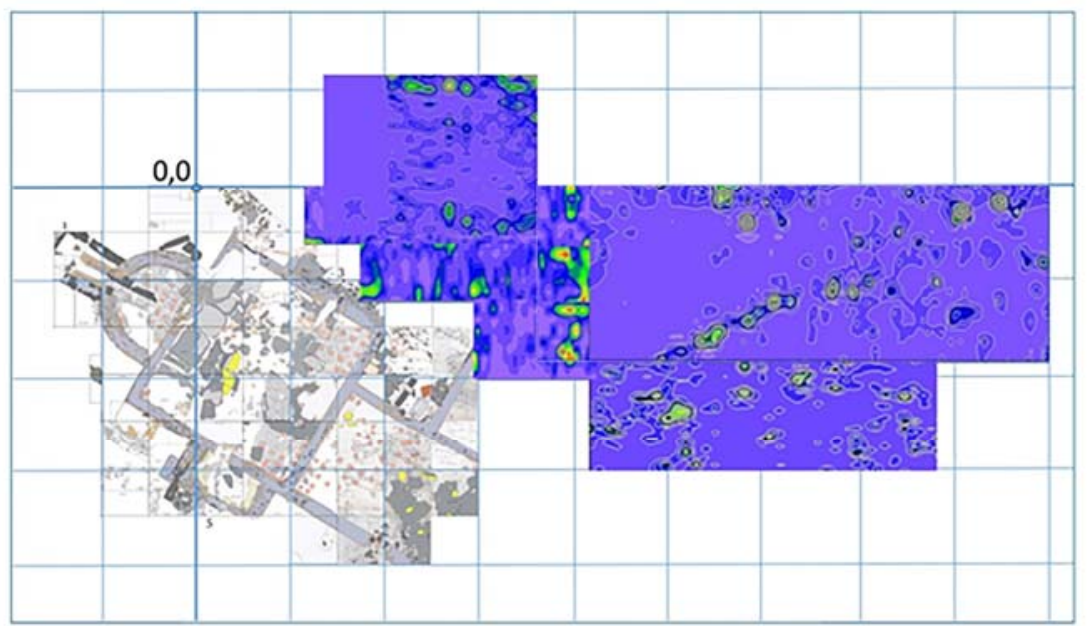

CarBath2

SLICE 4: 12-16 ns (96-128 cmbs)

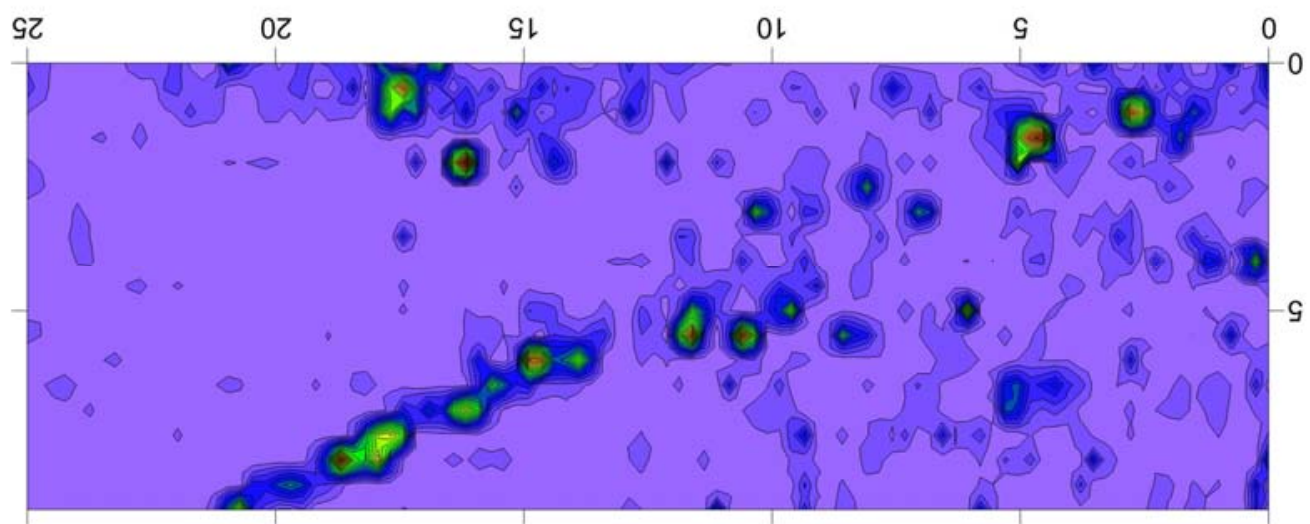



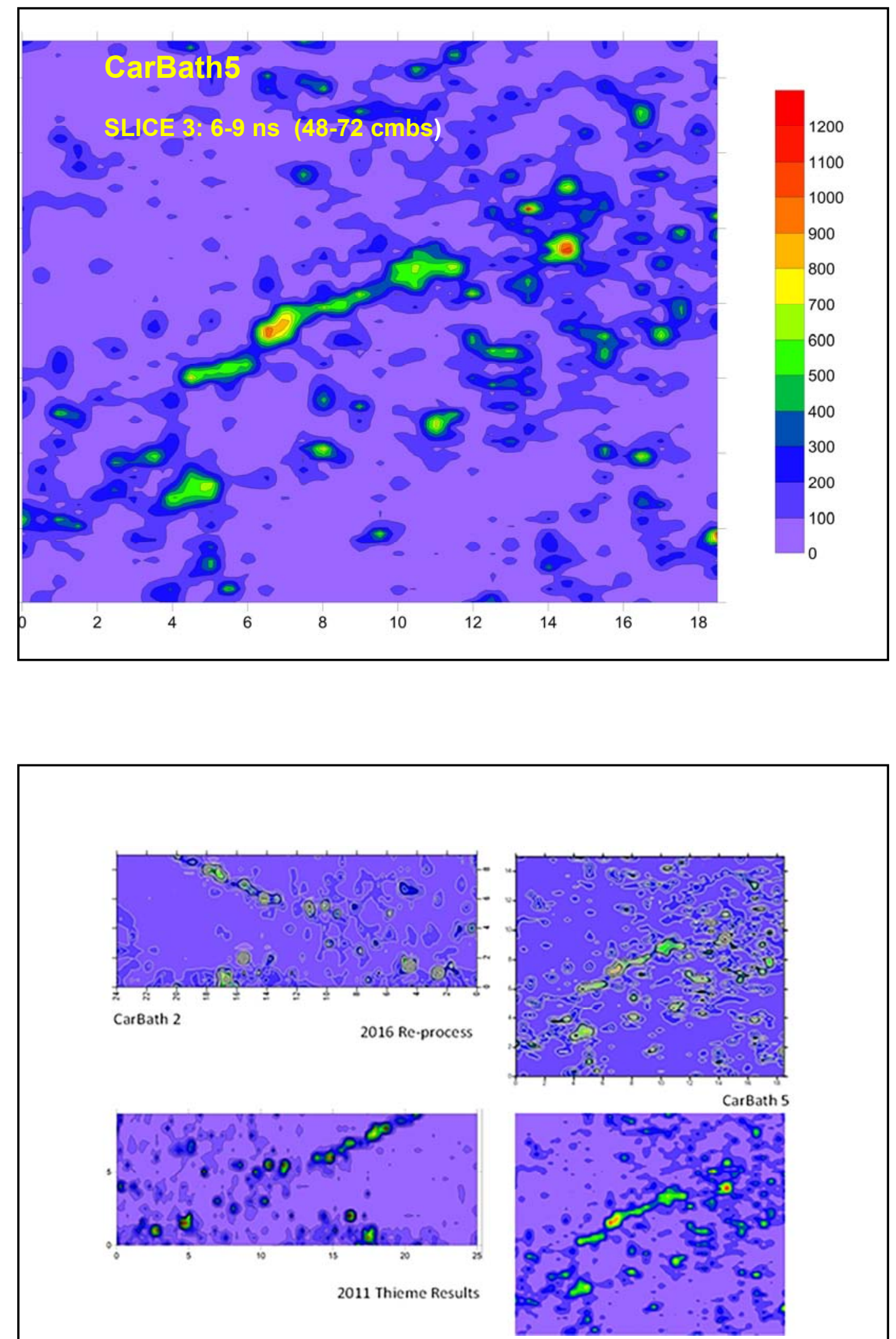


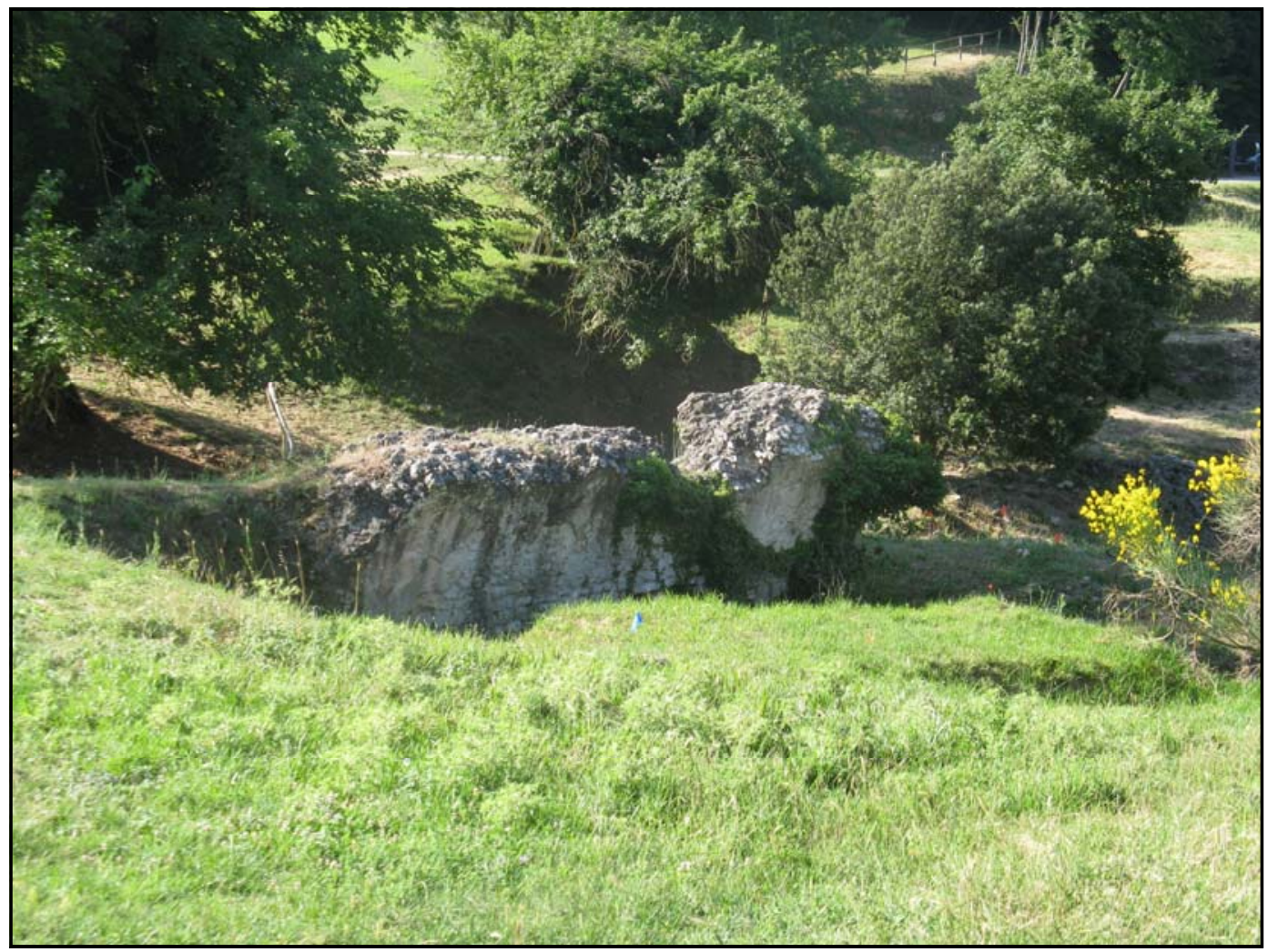

CarShed1

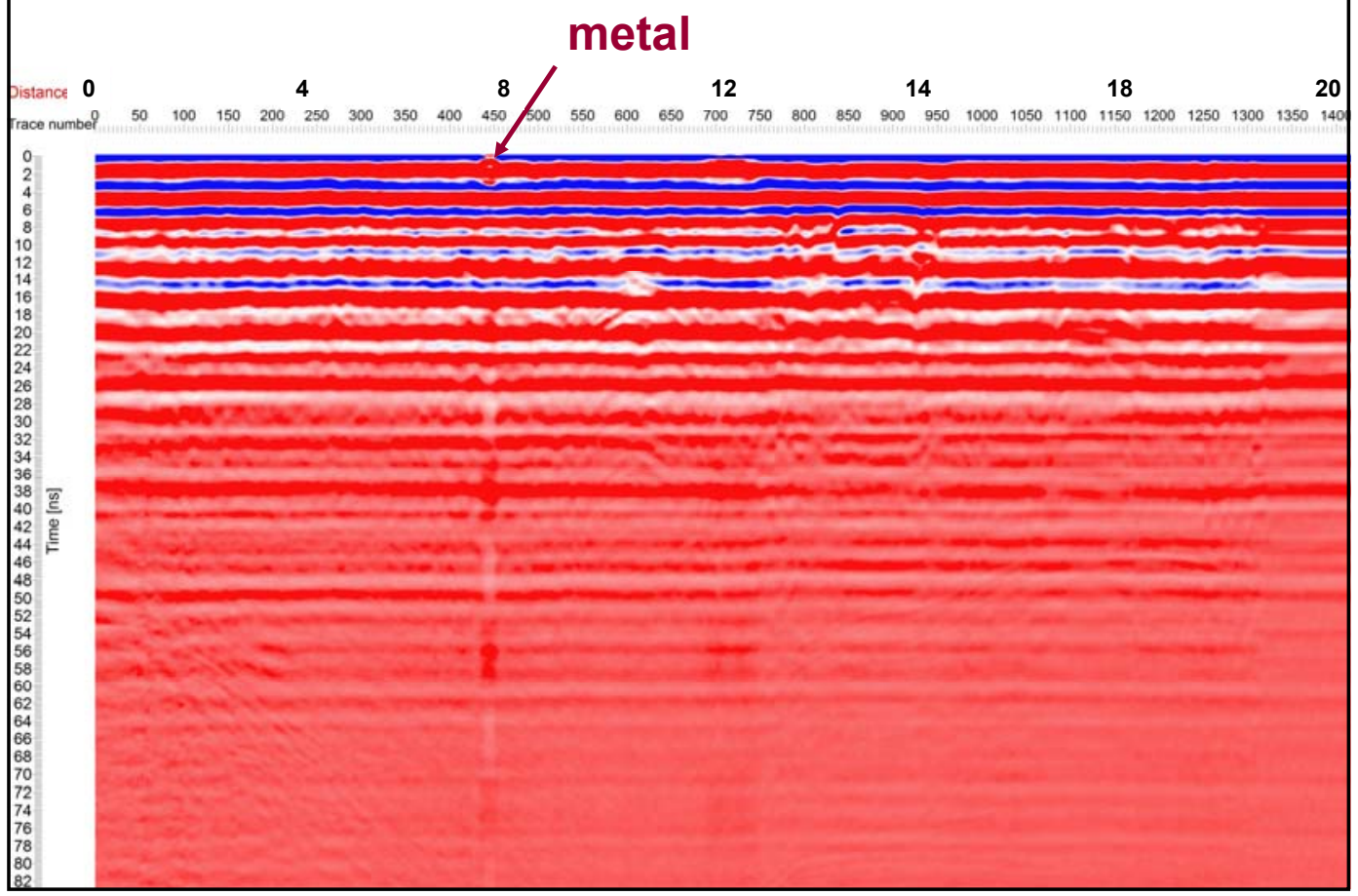




\section{CarShed1}
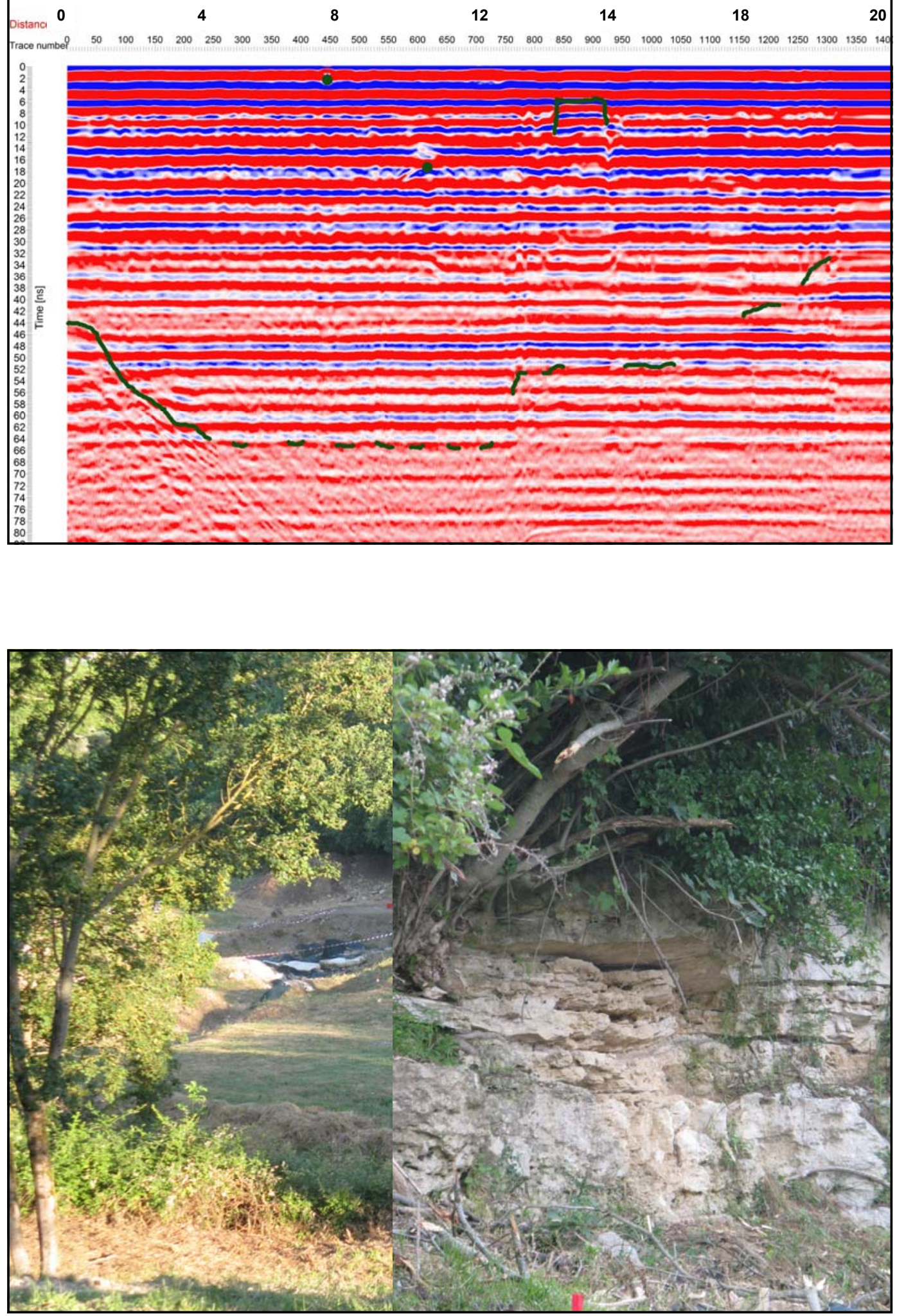


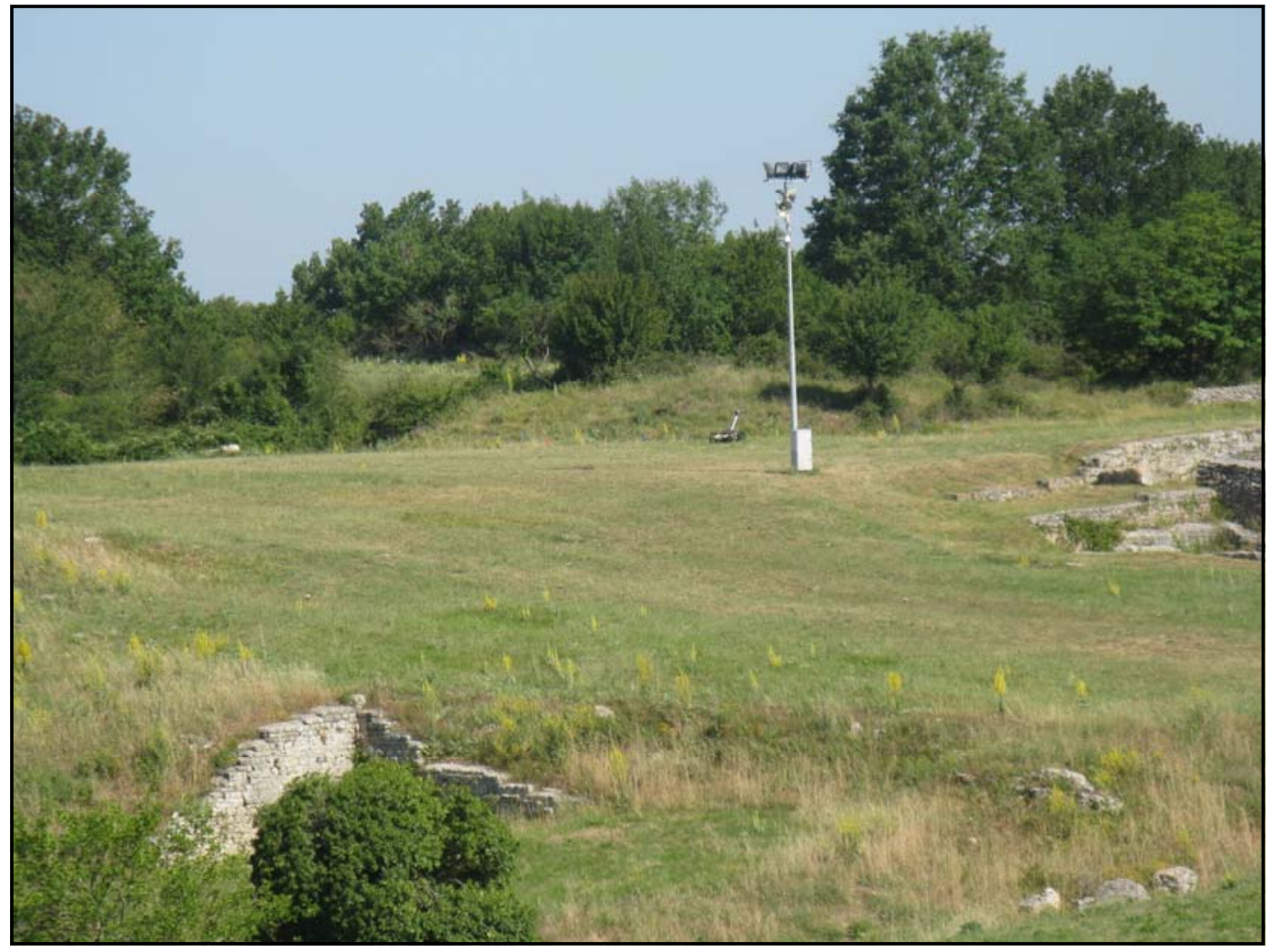

\section{Ciotti trench (Forum)}

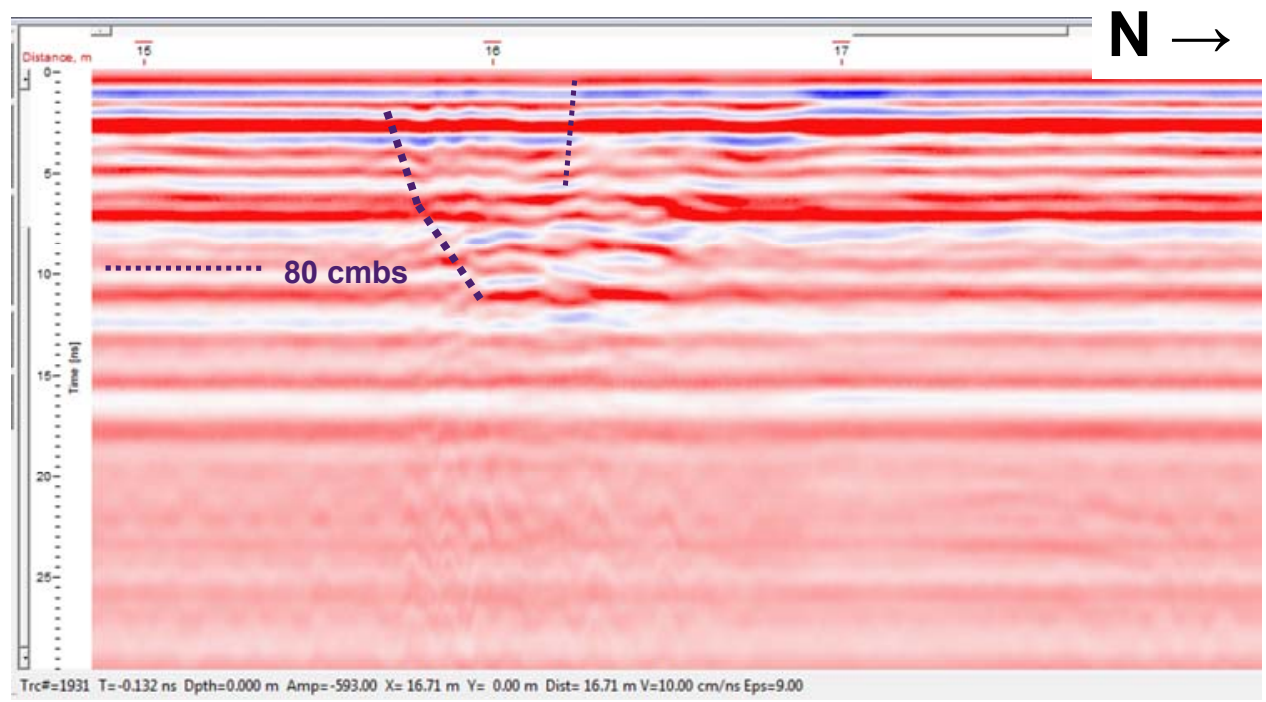




\section{Continuing Analysis}

- Processing in GPR-Slice of raw data files for all 13 Grids from Baths

- At least 2-3 slices at common levels

- Correct CarBath2 overlay where lower right corner was grid 0,0

- Correct possible offset in overlay of all grids on project excavation plan

- Match linear anomaly running from Cistern toward Baths with excavated features

\section{Continuing Analysis}

- Combine CarBathNS profile with seismological analysis of cultural/natural contact beneath Baths and fill in Fossa.

- Possible application in Carsulae Archaeological Park to identification of Ciotti excavations, looting, etc...

- Paleohydrology

- Paleoseismology 


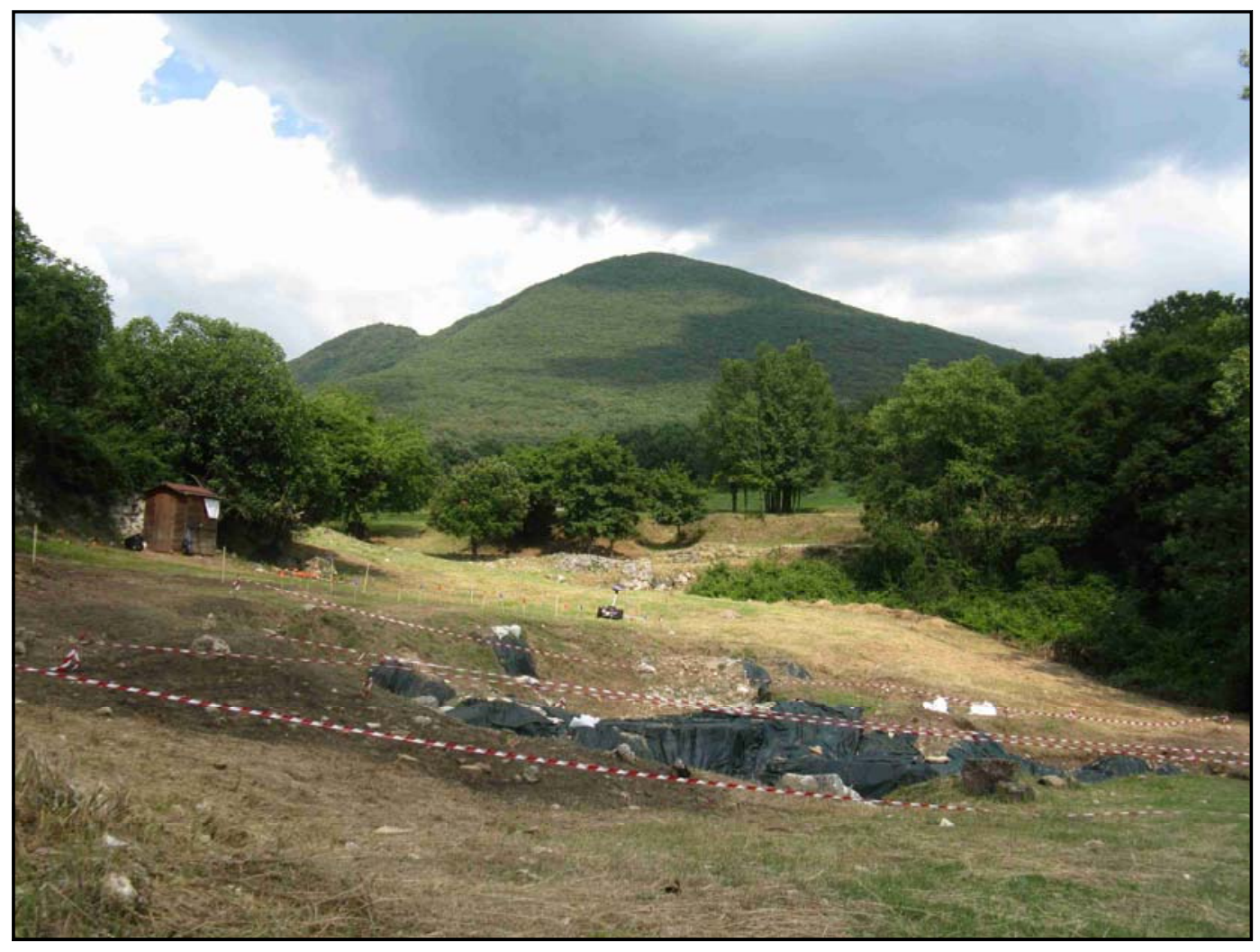

